Xiaojun Yu, MS*

Tingting $\mathrm{Du}, \mathrm{MS}^{*}$

Ning Song, $\mathrm{PhD}$

Qing $\mathrm{He}$, MS

Yong Shen, $\mathrm{PhD}$

Hong Jiang, $\mathrm{PhD}$

Junxia Xie, $\mathrm{PhD}$

Correspondence to

Dr. Jiang:

jhkyk@163.com or

Dr. Xie:

jxiaxie@public.qd.sd.cn

\section{Decreased iron levels in the temporal cortex in postmortem human brains with Parkinson disease}

ABSTRACT

Objective: The present study aimed to evaluate alterations in the levels of iron, divalent metal transporter 1 (DMT1) with the iron-responsive element (IRE), transferrin receptor 1 (TfR1), ferroportin 1 (FPN1), and iron regulatory protein 1 (IRP1) in the temporal cortex of human brains with Parkinson disease (PD).

Methods: Iron content was measured using an ICP-MS 7500CE detector. IRP1, DMT1+IRE, TfR1, and FPN1 expressions were determined by Western blotting.

Results: Iron content was significantly lower in the temporal cortex of patients with PD when compared with age-matched healthy controls. Unexpectedly, the levels of DMT1+IRE, TfR1, FPN1, and IRP1 were decreased in the temporal cortex in PD brains. No changes were observed in the temporal cortex of postmortem Alzheimer disease brains.

Conclusions: Iron deprivation and iron-related protein dysregulation suggest that a different iron regulatory mechanism may exist, and that iron redistribution may occur between the temporal cortex and the substantia nigra of patients with PD. Neurology ${ }^{\circledR}$ 2013;80:492-495

\section{GLOSSARY}

AD = Alzheimer disease; DMT1 = divalent metal transporter 1; FPN1 = ferroportin 1; HC = healthy controls; IRE $=$ ironresponsive element; IRP1 = iron regulatory protein 1; PD = Parkinson disease; SN = substantia nigra; TfR1 = transferrin receptor 1.

Postmortem studies have demonstrated that iron selectively accumulates in the substantia nigra (SN) of patients with Parkinson disease (PD). ${ }^{1}$ The dysregulation of iron transporters is thought to contribute to iron deposition in PD. Recently, increased expression of the iron importer divalent metal transporter 1 (DMT1) with the iron-responsive element (IRE, DMT1+IRE) has been reported in the SN of postmortem PD brains. ${ }^{2}$ Meanwhile, the downregulation of ferroportin 1 (FPN1), an iron exporter, was also suggested to be related to 6-hydroxydopamineinduced nigral iron accumulation. ${ }^{3}$

However, it is unknown whether iron metabolism is disrupted in brain regions other than the SN (e.g., the cerebral cortex) and how this occurs. In this study, we investigated iron content in the temporal cortex of postmortem PD brains. Unexpectedly, we observed decreased iron content in this brain region in PD but not in Alzheimer disease $(\mathrm{AD})$. To investigate the underlying mechanism, we further evaluated alterations in transporter expression, particularly DMT1+IRE, FPN1, and transferrin receptor 1 (TfR1). In PD brains, we observed decreased protein levels of DMT1+IRE, FPN1, and TfR1, and decreased expression of iron regulatory protein 1 (IRP1) in the cortex. These results imply that a different iron regulatory mechanism may exist in the temporal cortex, and that iron redistribution might occur between the cortex and SN in PD.

METHODS Subjects. The patients were recruited from Sun City, Arizona, a major suburb of Phoenix with a population of approximately 70,000. Brain tissue was obtained from the Banner Sun Health Research Institute Brain and Body Donation Program in Sun City,

\footnotetext{
*These authors contributed equally to this work.

From the Department of Physiology (X.Y., T.D., N.S., Q.H., H.J., J.X.), Shandong Provincial Key Laboratory of Pathogenesis and Prevention of Neurological Disorders and State Key Disciplines: Physiology, Medical College of Qingdao University, Qingdao, China; Center for Advanced Therapeutic Strategies for Brain Disorders (Y.S.), Roskamp Institute, Sarasota, FL; and Haldeman Laboratory of Molecular and Cellular Neurobiology (Y.S.), Sun Health Research Institute, Sun City, AZ.

Go to Neurology.org for full disclosures. Funding information and disclosures deemed relevant by the authors, if any, are provided at the end of the article.
} 
Arizona. ${ }^{4}$ The mean postmortem interval for this study was 2.6 hours. A clinical diagnosis was established according to the UK Parkinson's Disease Society Brain Bank clinical diagnostic criteria for PD, or the National Institute of Neurological and Communicative Disorders and Stroke criteria for AD. Healthy controls (HC) were selected based on age and no history of neuropathological diagnosis of neurologic or psychiatric disease. The HC were followed up clinically for 3 years to exclude the development of any neurodegenerative disease. The study population consisted of 10 cognitive HC subjects ( 4 male and 6 female; mean age $84.6 \pm 1.45$ years, range 78-92 years), 10 patients with $\mathrm{PD}$ (6 male and 4 female; mean age $82.7 \pm 1.65$ years, range $72-90$ years), and 10 patients with $\mathrm{AD}(3$ male and 7 female; mean age $84.1 \pm 1.84$ years, range 78-91 years).

Assessment of iron content. Temporal cortex tissue $(20 \mu \mathrm{g})$ was isolated from each brain and lysed with nitric acid. After adjusting the volume to $2 \mathrm{~mL}$, the levels of iron and other metals were measured using an ICP-MS 7500CE (Agilent, Santa Clara, $\mathrm{CA}$ ) inductively coupled plasma mass spectrometer.

Western blotting analysis. A total of $80 \mu \mathrm{g}$ of protein from each sample was separated using $8 \%$ sodium dodecyl sulfatepolyacrylamide gels and then transferred by electroblotting to polyvinylidene difluoride membranes. After 2 hours of blocking with $10 \%$ nonfat milk at room temperature, the membranes were incubated with primary antibody against DMT1+IRE, IRP1, TfR1, or FPN1 overnight at $4^{\circ} \mathrm{C}$, followed by incubation with secondary antibody conjugated to horseradish peroxidase. $\beta$-Actin was used as a loading control. Crossreactivity was visualized by enhanced chemiluminescence Western blotting detection reagents and then analyzed through scanning densitometry with a Tanon Image System (Tanon, Shanghai, China).

Standard protocol approvals, registration, and patient consent. Human tissues were collected with informed consent from subjects or next of kin and with ethical approval from the Banner Sun Health Institutional Review Board prior to the autopsy.

Statistical analysis. The data were presented as mean \pm SEM. A one-way analysis of variance followed by the Dunnett test was used to compare metal content between different groups. An unpairedsamples $t$ test was used to compare differences in protein levels. A probability value of $p<0.05$ was considered statistically significant.

RESULTS Iron content was lower in the temporal cortex of postmortem PD brains. Among the 4 metal ions measured, iron was abundant, but only trace amounts of the other 3 were detected. The iron levels in postmortem PD brains were much lower compared with those of $\mathrm{HC}$ (table). However, no changes were observed in the temporal cortex of postmortem $\mathrm{AD}$

\begin{tabular}{|c|c|c|c|c|}
\hline \multirow[t]{2}{*}{ Table } & \multicolumn{4}{|c|}{$\begin{array}{l}\text { Metal content in the temporal cortex of HC, patients with } P D \text {, and } \\
\text { patients with } A D \text { (ng/mg fresh weight of brain tissue) }{ }^{a}\end{array}$} \\
\hline & Mn & $\mathrm{Fe}$ & $\mathrm{Ni}$ & $\mathrm{Cu}$ \\
\hline $\mathrm{HC}$ & $1.23 \pm 0.22$ & $131.25 \pm 7.37$ & $1.51 \pm 0.70$ & $4.05 \pm 0.25$ \\
\hline PD & $0.87 \pm 0.09$ & $110.30 \pm 3.98^{b}$ & $0.35 \pm 0.12$ & $3.92 \pm 0.41$ \\
\hline$A D$ & $0.84 \pm 0.06$ & $123.07 \pm 9.65$ & $1.09 \pm 0.81$ & $4.86 \pm 1.62$ \\
\hline
\end{tabular}

Abbreviations: $A D=$ Alzheimer disease; $\mathrm{HC}=$ healthy controls; $\mathrm{PD}=$ Parkinson disease ${ }^{\text {a }}$ Data are presented as mean \pm SEM.

${ }^{\mathrm{b}}$ Analysis of variance test was used to compare group means; $p<0.05$ was considered as significant. brains. There was no difference for $\mathrm{Mn}, \mathrm{Ni}$, and $\mathrm{Cu}$ among the 3 groups.

Expression levels of DMT1+IRE, TfR1, FPN1, and IRP1 were decreased in the temporal cortex of PD brains. To investigate whether iron-related protein expression affected iron levels, we determined the expression levels of DMT1+IRE, TfR1, FPN1, and IRP1. We observed that these proteins were all downregulated in PD brains when compared with HC (figure). There was some overlap where some DMT+IRE and FPN1 levels were higher in a few PD brains than some control counterparts; however, on average there was a reduction in the levels in the PD cortex that showed statistical relevance. In PD brains, the protein levels were 22.22\% (DMT1), 14.23\% (TfR1), 21.11\% (FPN1), and 36.9\% (IRP1) lower than those in controls. No changes were observed in $\mathrm{AD}$ brains compared with $\mathrm{HC}$ (data not shown).

DISCUSSION The abnormal distribution of transition metal ions in specific brain regions has been reported in patients with neurodegenerative disorders, including $\mathrm{PD}$ and $\mathrm{AD}$. Although elevated iron has been previously examined extensively in $\mathrm{PD}$, we observed decreased iron levels in the temporal cortex of postmortem PD brains but not $\mathrm{AD}$ brains. However, this result was not consistent with a previous study, ${ }^{5}$ which reported no significant differences in the level of total iron in the cortex (Brodmann area 21) between parkinsonian patients and age-matched controls. This discrepancy between the 2 studies might be due to the different measurement methods, severity stages, and race. Because iron misdistribution might be involved in several different disorders, ${ }^{6}$ we supposed that iron redistribution might exist in $\mathrm{PD}$ brains, particularly between the temporal cortex and the SN.

Several proteins responsible for intracellular iron homeostasis might be involved in iron metabolism disruption in the cerebral cortex. We first focused on DMT1+IRE, which possesses an IRE sequence in the $3^{\prime}$-untranslated region and is responsive to iron through an IRE/IRP-dependent mechanism. Normally when the intracellular iron level decreased, DMT1+IRE was upregulated by enhanced IRP1 binding to the IRE to maintain iron homeostasis. However, this regulatory mechanism obviously failed to explain our findings that both iron and IRP1/ DMT1+IRE levels were decreased in the temporal cortex of PD brains. Therefore, DMT1+IRE downregulation might be the primary cause of the iron deprivation observed in the PD temporal cortex and not a secondary event. Contrary to an unchanged IRP1 binding activity found in the $\mathrm{SN}$ of $\mathrm{PD}$ brains, ${ }^{7}$ we supposed that with a lower IRP1 protein level, the affinity of IRP1 binding to the IRE must be impaired, eventually leading to decreased DMT1+IRE and TfR1 expression in the temporal cortex of PD brains. 
Figure Expression levels of divalent metal transporter 1 with iron-responsive element, transferrin receptor 1 , ferroportin 1, and iron regulatory protein 1 in the temporal cortex of postmortem Parkinson disease brains and age-matched controls

A
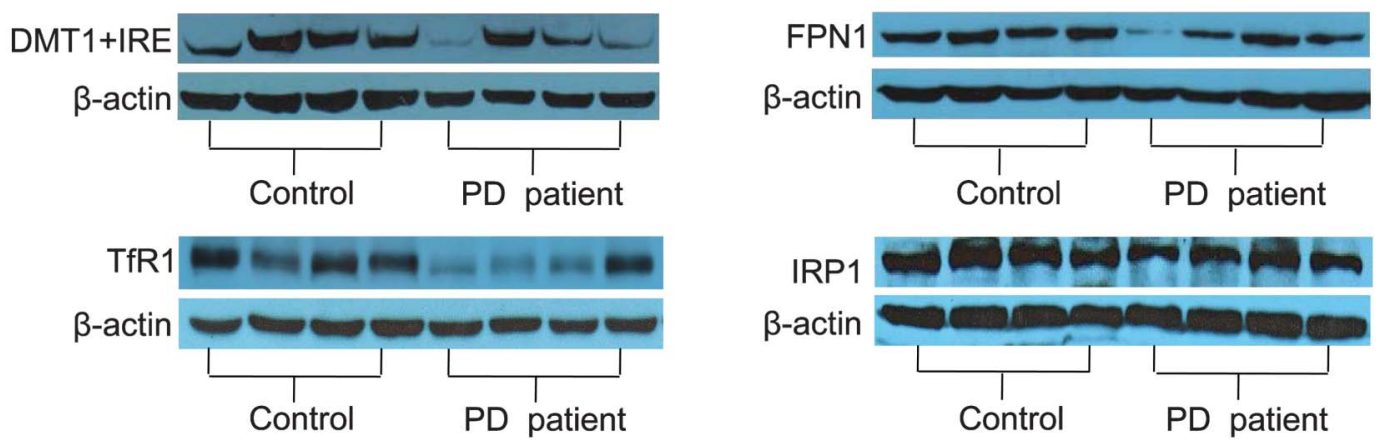

B

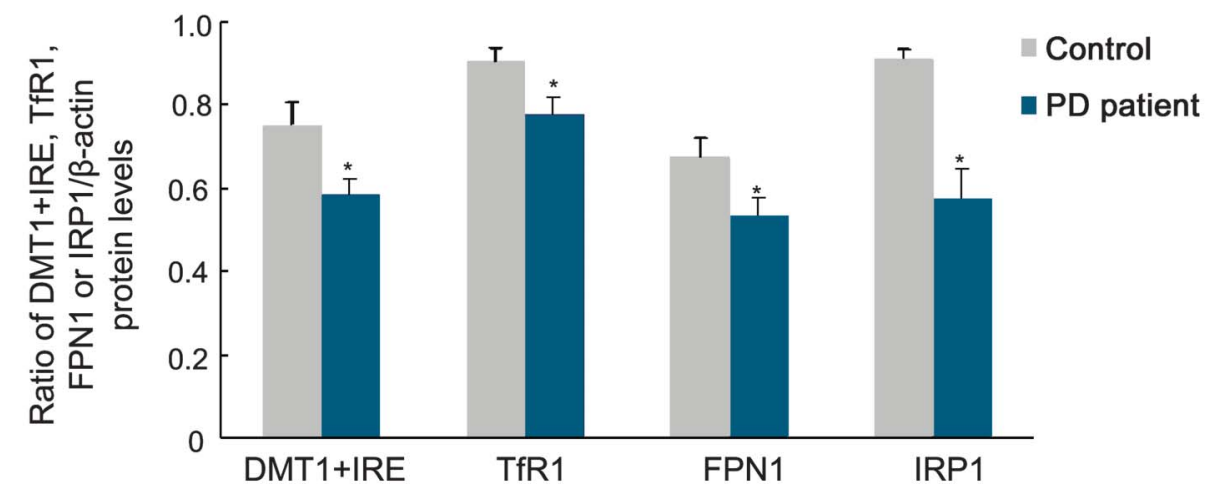

(A) Divalent metal transporter 1 (DMT1) with iron-responsive element (IRE, + IRE), transferrin receptor 1 (TfR1), ferroportin 1 (FPN1), and iron regulatory protein 1 (IRP1) expressions were detected by Western blotting at 55, 95, 62, or $98 \mathrm{kDa}$, respectively, using antibodies against these proteins. $\beta$-Actin was used as a loading control. (B) Scanning densitometric quantification of protein expression. DMT1+IRE, TfR1, FPN1, and IRP1 expressions were decreased in the Parkinson disease group relative to age-matched controls. Data are presented as the ratios of DMT1+IRE, TfR1, FPN1, or IRP1 to $\beta$-actin. ${ }^{*} p<0.05$, compared with control.

Our previous studies showing that DMT1+IRE downregulation could be initiated by IRP1 knockdown may further confirm this notion. ${ }^{8}$ Abnormal cerebral perfusion occurs in PD. ${ }^{9}$ Hypoxia and other factors have been reported to suppress IRP/IRE binding affinity and to induce the downregulation of IRPs. ${ }^{10}$ We believe as an iron exporter, even FPN1 downregulation was a compensatory mechanism to partially offset reduced DMT1+IRE and iron import; however, this might fail to counteract the preceding process of iron deprivation.

In this study, we showed that the downregulation of DMTI+IRE, TfR1, FPN1, and IRP1 contributed to decreased iron content in the temporal cortex of PD brains. These findings also suggest that iron homeostasis is disrupted through different mechanisms in the $\mathrm{SN}$ and temporal cortex.

\section{AUTHOR CONTRIBUTIONS}

X. Yu performed Western blots. T. Du determined metal content. N. Song analyzed data. Q. He determined metal content. Y. Shen supplied brain samples and participated in the experiment design. H. Jiang participated in the experiment design, analyzed data, and drafted the manuscript. J. Xie participated in the experiment design and drafted the manuscript.

\section{STUDY FUNDING}

Supported by grants from the National Program of Basic Research sponsored by the National Foundation of Natural Science of China (30930036, 30900477, 81171207), the Ministry of Science and Technology of China (2011CB504102, 2012CB526703), and Fok Ying Tong Education Foundation (121034). Also supported in part by NIHRO1AG025888. Sun Health Research Institute Brain and Body Donation Program of Sun City, Arizona, provided human biological materials (or specific description, e.g., brain tissue). The Brain and Body Donation Program is supported by the National Institute of Neurological Disorders and Stroke (U24 NS072026 National Brain and Tissue Resource for Parkinson's Disease and Related Disorders), the National Institute on Aging (P30 AG19610 Arizona Alzheimer's Disease Core Center), the Arizona Department of Health Services (contract 211002, Arizona Alzheimer's Research Center), the Arizona Biomedical Research Commission (contracts 4001, 0011, 05-901, and 1001 to the Arizona Parkinson's Disease Consortium), and the Michael J. Fox Foundation for Parkinson's Research.

\section{DISCLOSURE}

X. Yu and T. Du report no disclosures. N. Song received research support from National Natural Science Foundation of China (30900477). Q. He reports no disclosures. Y. Shen received research support from National Institutes of Health/National Institute on Aging. H. Jiang received research supports from 973 program (2012CB526703), National Natural 
Science Foundation of China (81171207), and Fok Ying Tong Education Foundation (121034). J. Xie received research supports from 973 program (2011CB504102) and National Natural Science Foundation of China (30930036). Go to Neurology.org for full disclosures.

Received March 25, 2012. Accepted in final form September 24, 2012.

\section{REFERENCES}

1. Sofic E, Paulus W, Jellinger K, Riederer P, Youdim MB. Selective increase of iron in substantia nigra zona compacta of parkinsonian brains. J Neurochem 1991;56:978-982.

2. Salazar J, Mena N, Hunot S, et al. Divalent metal transporter 1 (DMT1) contributes to neurodegeneration in animal models of Parkinson's disease. Proc Natl Acad Sci USA 2008;105: 18578-18583.

3. Wang J, Jiang H, Xie JX. Ferroportin1 and hephaestin are involved in the nigral iron accumulation of 6-OHDAlesioned rats. Eur J Neurosci 2007;25:2766-2772.

4. Beach TG, Sue LI, Walker DG, et al. The Sun Health Research Institute Brain Donation Program: description and experience, 1987-2007. Cell Tissue Bank 2008;9:229-245.
5. Sofic E, Riederer P, Heinsen H, et al. Increased iron (III) and total iron content in post mortem substantia nigra of parkinsonian brain. J Neural Transm 1988;74:199-205.

6. Kakhlon O, Breuer W, Munnich A, Cabantchik ZI. Iron redistribution as a therapeutic strategy for treating diseases of localized iron accumulation. Can J Physiol Pharmacol 2010;88:187-196.

7. Faucheux BA, Martin ME, Beaumont C, et al. Lack of upregulation of ferritin is associated with sustained iron regulatory protein-1 binding activity in the substantia nigra of patients with Parkinson's disease. J Neurochem 2002;83: 320-330.

8. Jiang H, Song N, Xu H, Zhang S, Wang J, Xie J. Upregulation of divalent metal transporter 1 in 6-hydroxydopamine intoxication is IRE/IRP dependent. Cell Res 2010;20:345-356.

9. Melzer TR, Watts R, MacAskill MR, et al. Arterial spin labelling reveals an abnormal cerebral perfusion pattern in Parkinson's disease. Brain 2011;134:845-855.

10. Recalcati S, Minotti G, Cairo G. Iron regulatory proteins: from molecular mechanisms to drug development. Antioxid Redox Signal 2010;13:1593-1616.

\section{Target Your Job Search}

Your goal is precise, your time is precious. So give it your best shot.

The AAN's Neurology Career Center is the largest neurology-specific job site tailored to in-demand neurology professionals like you.

Visit www.aan.com/careers and create your free profile today.

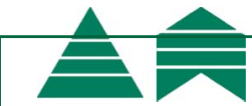

\section{Editor's Note to Authors and Readers: Levels of Evidence in Neurology ${ }^{\circledR}$}

Effective January 15, 2009, authors submitting Articles or Clinical/Scientific Notes to Neurology ${ }^{(B)}$ that report on clinical therapeutic studies must state the study type, the primary research question(s), and the classification of level of evidence assigned to each question based on the AAN classification scheme requirements. While the authors will initially assign a level of evidence, the final level will be adjudicated by an independent team prior to publication. Ultimately, these levels can be translated into classes of recommendations for clinical care. For more information, please access the articles and the editorial on the use of classification of levels of evidence published in Neurology. ${ }^{1-3}$

1. French J, Gronseth G. Lost in a jungle of evidence: we need a compass. Neurology 2008;71:1634-1638.

2. Gronseth G, French J. Practice parameters and technology assessments: what they are, what they are not, and why you should care. Neurology 2008;71:1639-1643.

3. Gross RA, Johnston KC. Levels of evidence: taking Neurology ${ }^{\circledR}$ to the next level. Neurology 2009;72:8-10. 